\section{Alimentäre Allergieprävention mit stark hydrolysierter Säuglingsnahrung}

\author{
Kinder mit familiärer Atopiebelastung sollten, falls sie nicht gestillt \\ werden können, eine stark hydrolysierte Formelnahrung erhalten.
}

$\mathrm{D}$ as Allergierisiko wird durch die Interaktion von genetischen und umweltbedingten Faktoren beeinflußt. Allergieprävention sollte daher in erster Linie für Kinder aus Allergiker-Familien angestrebt werden. Als Präventionsmaßnahmen werden Stillen und das Meiden von Inhalations- und Nahrungsmittelallergenen empfohlen. Unklar ist jedoch noch immer, ob in der Allergieprävention stark hydrolysierte Säuglingsnahrung effizienter ist als eine partiell hydrolysierte Formula.

\section{Drei Formula im Vergleich}

Im Rahmen einer prospektiven, randomisierten Blindstudie wurde dieser Frage jetzt nachgegangen. Die schwedischen Wissenschaftler untersuchten 162 Kinder mit familiärer Atopiebelastung. Eine Woche vor dem erwarteten Geburtstermin eliminierten die werdenden Mütter alle Nahrungsmittel, die Kuhmilch, Hühnerei und Fisch enthielten, aus ihrer Ernährung. Diese Eliminationsdiät wurde während der gesamten Stilldauer unter Kalziumsupplementierung beibehalten.

$\mathrm{Zu}$ Beginn des Abstillens wurden die Kinder randomisiert einer von drei Formula-Gruppen zugeordnet: 50 Kinder erhielten eine stark hydrolysierte Kasein-Formula (Nutramigen ${ }^{\circledR}$, Mead Johnson), 45 eine partiell hydrolysierte Molken-Kasein-Formula (Mead Johnson) und 46 eine Muttermilch-adaptierte Routine-Formula (Enfamil ${ }^{\circledR}$, Mead Johnson). Weitere 21 Kinder wurden neun Monate oder länger voll gestillt. Beikost wurde frühestens nach dem 4. Lebensmonat, während der Formula-Ernährungsphase ohne milchhaltige Nahrungsmittel und innerhalb des 1. Lebensjahres ohne Ei, Fisch und Zitrusfrüchte eingeführt. Alle Kinder wurden im Alter von 3, 6, 9, 12 und 18 Monaten auf Anzeichen von atopischen Er- krankungen hin untersucht. Überdies wurden Hautpricktests durchgeführt und nach allergenspezifischen IgEAntikörpern gesucht.

\section{Geringere Allergieprävalenz unter der stark hydrolysierten Formula}

Die kumulative Inzidenz atopischer Symptome war in der Gruppe mit stark hydrolysierter Formula nach 6, 9, 12 und 18 Monaten signifikant niedriger als in der Routine-Formula-Gruppe. Auch im Vergleich zur Gruppe mit partiellem Hydrolysat zeigte die Gruppe mit extensivem Hydrolysat nach 6 und 9 Monaten signifikant weniger atopische Symptome. Nach 18 Monaten waren bei $51 \%$ der Kinder
D ie alimentäre Atopieprävention wird seit Jahren kontrovers diskutiert. Ein genereller präventiver Nutzen allergenarmer Diäten während der Schwangerschaft und der Stillzeit konnte bisher nicht dokumentiert werden. Ob hypoallergene Formelnahrung in Abhängigkeit ihres Hydrolysegrades einen unterschiedlichen atopiepräventiven Effekt hat, wurde erstmals vergleichend von der schwedischen Forschergruppe in einer sorgfältig durchgeführten Studie untersucht. Sie zeigt, daß atopische Hochrisikokinder nach dem Abstillen nur von einer stark hydrolysierten Kasein-Formula profitieren und nicht von einer partiell hydrolysierten Formelnahrung.

Zur abschließenden Bewertung der allergiepräventiven Potentiale stark hydrolysierter Formelnahrung und zur Klärung der langfristigen Auswirkungen auf den atopischen Krankheitsverlauf bedarf es jedoch unter extensivem Hydrolysat, 64\% der Gruppe mit partiellem Hydrolysat, $84 \%$ der Routine-Formula-Gruppe und $67 \%$ der voll gestillten Kinder atopische Symptome aufgetreten.

Im Alter von 9 Monaten hatten signifikant weniger Kinder der Gruppe mit extensivem Hydrolysat einen positiven Hautpricktest auf Ei als in der Gruppe mit partiellem Hydrolysat. Die allergenspezifischen IgE-Antikörper der vier Gruppen unterschieden sich jedoch nicht signifikant.

Insgesamt konnte gezeigt werden, daß signifikante atopiepräventive Effekte bei Hochrisikokindern nach dem Abstillen nur mit einer stark hydrolysierten Kasein-Formula zu erwarten sind.

Quelle: Oldaeus G, Anjou K, Björksten B, et al.: Extensively and partially hydrolysed infant formulas for allergy prophylaxis. Arch Dis Child (1997); 77:4-10.

Dr. med. R. Kehrt, Klinik für Pädiatrie, Virchow-Klinikum, Universitätsklinik Berlin, Augustenburger Platz 1, D-13353 Berlin.

\title{
Kommentar
}

weiterer vergleichender Studien. Die in Deutschland multizentrisch durchgeführte Untersuchung zum Einfluß der Säuglingsernährung auf die Entstehung einer Allergie (GINI-Studie) läßt aussagekräftige Ergebnisse erwarten.

Ein bis zum jungen Erwachsenenalter anhaltender allergiepräventiver Effekt ist bislang nur für Muttermilch beschrieben. So bleibt die Empfehlung, möglichst alle atopischen Hochrisikokinder konsequent sechs Monate voll zu stillen und Beikost im ersten Lebensjahr hühnereiweißfrei einzuführen, weiterhin gültig. Für Kinder, die nicht gestillt werden können, empfiehlt sich eine stark hydrolysierte Formelnahrung, auch vor dem Einschießen der Muttermilch.

Bis zur weiteren Erforschung der Toleranzinduktion kann daher Allergieprävention nur Allergenreduktion bedeuten.

Rainer Kehrt 